\title{
Vers une nomenclature et un schéma dosimétrique applicables à toutes les radiations $\left(^{*}\right)$
}

\author{
C. S. RUPERT $\left({ }^{* *}\right)$ et R. LATARJET $(* * *)$ \\ (Manuscrit reçu le 2 février 1979)
}

\begin{abstract}
RÉSUMÉ
Cet article décrit brièvement les conclusions d'un groupe international de travail qui s'est réuni à Sèvres en avril 1975 dans le but d'harmoniser les conceptions sur les grandeurs destinées à définir un champ de radiation.
\end{abstract}

\section{SUMMARY}

This study summarises the conclusions of a Joint Working Group on Radiation Quantities, which met at Sèvres in April 1975 with the goal of harmonizing the concepts about those quantities which describe a radiation field.

Les radiations - considérées comme un transfert d'énergie d'un point à un autre sans dépendance d'un milieu matériel - se présentent sous des aspects divers. Certains de ces aspects sont couramment décrits comme des faisceaux de particules (tels que des faisceaux d'électrons ou de neutrons); d'autres comme des trains d'ondes (tels des rayonnements électromagnétiques); mais chacune de ces deux descriptions peut être employée pour n'importe quel type de radiation. Ceux qui travaillent avec les rayonnements visibles et ultraviolets sont bien habitués aux deux terminologies, ondulatoire et particulaire (photon), et utilisent celle qui convient le mieux au problème en cours.

En dépit de particularités individuelles, tous les types de radiation ont des caractères communs, et leurs similitudes sont encore plus grandes lorsqu'on considère seulement une gamme limitée de phénomènes au plan pratique. Aussi, pour de nombreux motifs intéressant les photochimistes et les photo-

(*) Cet article est la rédaction en français effectuée par l'un des auteurs (R.L.) d'un article publié en Anglais dans Photochemistry et Photobiology, 1978, t. 28, p. 3. Nous remercions l'éditeur de ce journal, M. PILL SOON SoNG, de nous en avoir donné l'autorisation.

(**) The University of Texas at Dallas, Richardson, Texas 75080, U.S.A.

(***) Institut Curie, 26, rue d'Ulm, 75005 Paris, France.

RADIOPROTECTION, VOL. 14 - 0033-8451/1979/117/\$ 4.00/C Bordas-Dunod. 
biologistes $\left({ }^{1}\right)$, tout champ d'une radiation incohérente et non polarisée peut être simplement décrit comme un flux d'énergie ou de particules en trois dimensions. Ses interactions avec la matière peuvent être prédites si l'on dispose de suffisamment de données sur le flux ainsi que sur la valeur des coefficients propres à l'interaction.

Les modifications radiochimiques et photochimiques induites par les radiations, ainsi que les phénomènes biologiques qui en découlent, prennent leur origine dans de telles interactions d'un champ de radiation et de la matière. Comme tous les types de champs de radiation peuvent être décrits de la même façon, il semble qu'un seul ensemble de concepts dosimétriques devrait pouvoir être employé pour tous. Malheureusement, l'histoire s'est déroulée autrement. Pour chaque type de radiation, les premiers expérimentateurs eurent affaire à des problèmes particuliers différents. Il en a résulté une diversité de définitions et de concepts qui constituent non seulement un obstacle à la communication scientifique, mais aussi une gêne pour les agences responsables des problèmes de radioprotection.

Au cours du Congrès International de Recherche sur les Radiations à Seattle en 1974, des représentants de trois organisations internationales : Commission Internationale de l'Éclairage (C.I.E.); Association Internationale de Photobiologie (A.I.P.); Commission Internationale des Unités et Mesures de Radiation (I.C.R.U.), constituèrent un groupe de travail informel chargé d'étudier et de recommander à leurs organisations un schéma dosimétrique cohérent. Ce groupe fut complété par les représentants de l'Union Radioscientifique Internationale (U.R.S.I.). Il tint sa première réunion en avril 1975 au Bureau International des Poids et Mesures à Sèvres $\left({ }^{2}\right)$.

Cette réunion (qui devrait être suivie d'autres) fut entièrement consacrée aux grandeurs nécessaires à la description d'un champ de radiation. Elle fut donc restreinte à la considération des rayonnements qui se propagent (avant toute interaction avec une cible, donc avant qu'ils deviennent intéressants pour l'utilisateur). Les conclusions, que nous résumons ici brièvement, sont encore à l'étude auprès de chaque organisation membre, et c'est seulement lorsque leur accord sera réalisé à ce niveau que le groupe poursuivra ses travaux.

Le faisceau de particules ou d'énergie au voisinage d'un point est distribué en espace, en direction, en temps, et, finalement, en énergie (énergie de particules ou longueurs d'ondes). Comme toutes les fonctions de distribution sont finies, tout élément du faisceau occupe un certain angle solide $d \Omega$, une surface de section $d a$ perpendiculaire à la direction de propagation, une durée $d t$

(1) C'est à ceux-ci que les auteurs s'adressent ici; mais les présentes considérations sont valables également pour ceux qui s'intéressent aux radiations ionisantes (note du présent éditeur).

${ }^{2}$ ) Les participants étaient : Président : J. Terrien, Directeur du Bureau; Délégués de l'A.I.P. : R. LAtARJet et C. S. Rupert; Délégués de l'I.C.R.U. : A. Allisy et H. O. WyCKoff; Délégués de la C.I.E. : J. Dourgnon et J. TERRIEN; Délégué de l'U.R.S.I. : P. Weissglas. Autres participants : H. S. BoYne, U.S. Natl. Bureau of Standards; P. Giacomo, B.I.P.M. et J. Chavaudra, Institut Gustave-Roussy, Villejuif. 


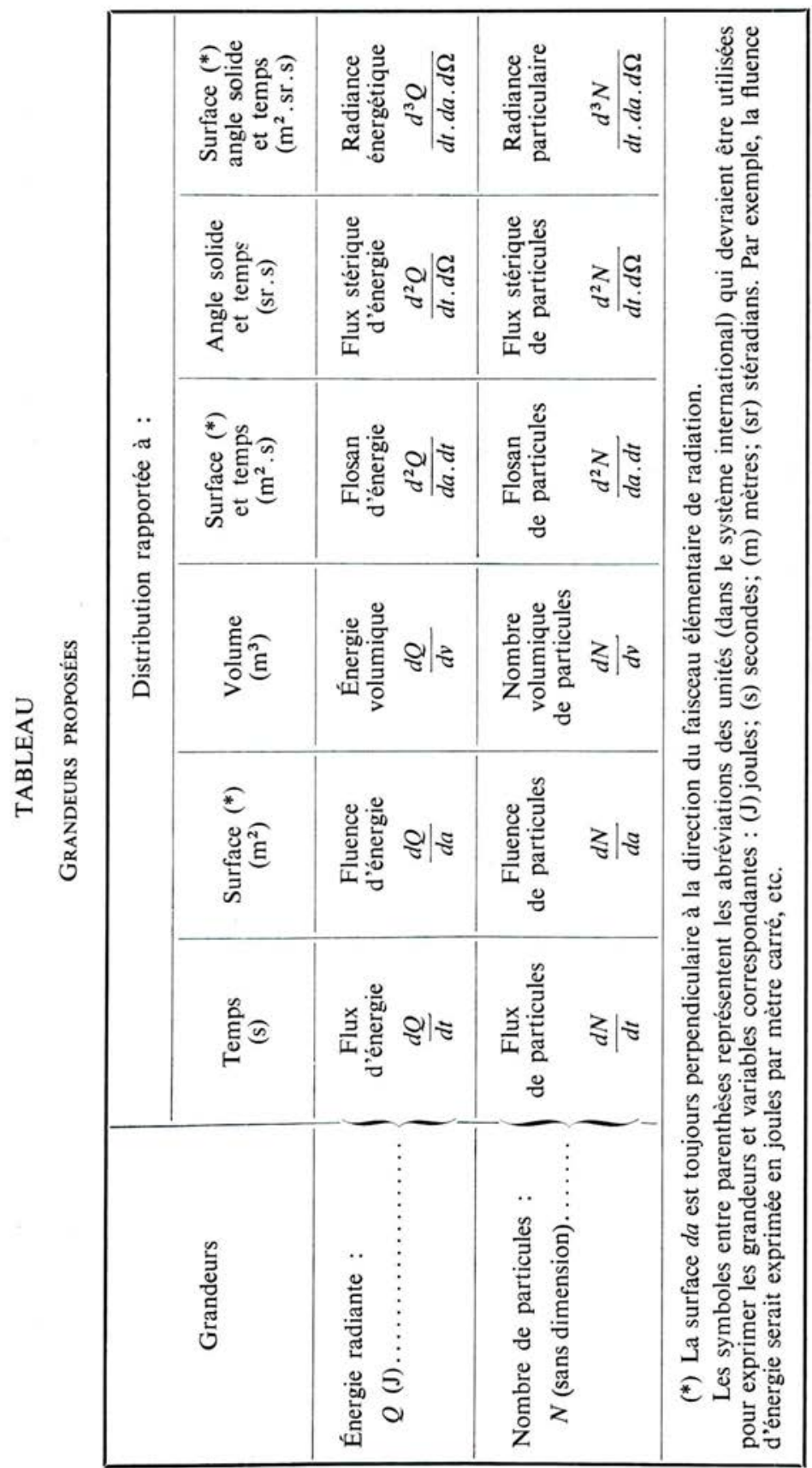


et une gamme d'énergies de particules $d E$ (ou de fréquences $d v$ ). La plupart des caractéristiques importantes du champ de radiation sont fixées si les fonctions de distribution concernant ces variables sont spécifiées. Ces fonctions sont donc les premières quantités susceptibles de décrire le champ. Les plus importantes d'entre elles sont résumées dans le tableau avec les noms que le groupe de travail a recommandés (des problèmes demeurent quant aux symboles, et plusieurs de ceux qui figurent sur le tableau pourraient ne pas être les choix définitifs).

La première grandeur intéressante est la quantité totale de l'énergie transportée par la radiation, l'énergie radiante (tableau, colonne 1), exprimée en joules (J). Si la radiation est considérée comme faite de particules avec des énergies définies, une grandeur équivalente est le nombre total des particules (c'est-à-dire, ici, des photons). Cette dernière grandeur est sans dimension, mais les mêmes fonctions de distribution sont utiles dans les deux cas, aboutissant à un parallélisme complet des grandeurs de champ définies.

La quantité d'énergie transportée par la radiation par unité de temps est le flux d'énergie décrit par la fonction $d Q / d t$ (colonne 2) et exprimée en watts $\left(\mathrm{J} \cdot \mathrm{s}^{-1}\right)$. La quantité parallèle est le flux de particules (ou, ici, le flux de photons) exprimé en nombre de particules par seconde.

A chaque instant et en chaque lieu existe une certaine densité spatiale de l'énergie ou de particules, soit en volume $d / d v$, soit en surface $d / d a$. La densité sur une surface perpendiculaire à la direction de propagation présente un intérêt spécial pour la description du champ.

Pour la première de ces descriptions le terme d'énergie volumique (exprimée en $\mathbf{J} . \mathrm{m}^{-3}$ ) est recommandé dans les cas où la radiation est décrite en termes d'énergie (colonne 4). Toutefois, l'absence d'un mot pour désigner le « nombre de particules " nous empêche de donner un terme équivalent si l'on parle en termes de photons.

Pour la distribution sur une surface perpendiculaire à la direction de propagation, les termes de fluence d'énergie et de fluence de particules (colonne 3), déjà adoptés par l'I.C.R.U., ont été retenus en dépit des objections qui ont été formulées au sein du groupe de travail $\left({ }^{1}\right)$.

Ces grandeurs, à savoir la quantité d'énergie par unité de surface $\left({\left.\mathrm{J} . \mathrm{m}^{-2}\right)}^{2}\right.$ ou le nombre de photons par unité de surface $\left(\mathrm{m}^{-2}\right)$ qui ont traversé une surface perpendiculaire à la direction du faisceau, sont particulièrement intéressantes en photobiologie ultraviolette. Dans une région où la radiation vient de plusieurs directions différentes, la description demande plus de soin. L'analyse montre (voir par exemple [1]) que, dans ce cas, la grandeur est l'énergie (ou le nombre de particules) par unité de surface perpendiculaire à chaque direction du faisceau contributeur; ces énergies sont additionnées pour toutes les directions qui composent l'ensemble. Un tel calcul équivaut à additionner toutes les énergies (ou les particules) qui pénètrent de toutes

( $\left.{ }^{1}\right)$ L'un de nous (R. L.) a formulé les plus expresses réserves sur cette terminologie (note du présent éditeur). 
les directions dans une petite sphère centrée sur le lieu considéré, et à diviser cette somme par la section de la sphère. On doit noter que la grandeur $\left({ }^{1}\right.$ ) résultante n'est pas égale à l'énergie, ou aux particules, par unité de surface traversant chaque surface particulière, à moins que la radiation ne provienne en grande majorité que d'une seule direction.

Lorsque la vitesse de propagation est connue, l'énergie volumique (ou le nombre volumique de particules) peut être calculée à partir du débit de la fluence dans le temps et vice versa. Laquelle de ces grandeurs est la plus naturelle dépend entièrement du problème particulier et du type d'interaction avec la matière qui sont considérés. Ce choix ne nous concerne pas ici puisque, pour décrire un champ de radiation en un point donné, ces deux densités sont équivalentes.

Le débit de la fluence dans le temps intervient fréquemment dans la description des champs. Ceci rend donc désirable un nom particulier pour la distribution correspondante. C'est pourquoi l'acronyme flosan a été formé ( $f$ lux over surface area normal), terme sans précédent dans le langage scientifique, et qui implique seulement sa propre définition. Un champ peut être décrit soit en termes de flosans d'énergie (exprimés en $\mathrm{J} \cdot \mathrm{m}^{-2} \cdot \mathrm{s}^{-1}$ ), soit en flosans de particules (exprimés en $\mathrm{m}^{-2} \cdot \mathrm{s}^{-1}$ ).

Le flux stérique d'énergie (colonne 5) exprimé en joules par seconde et par stéradian $\left(\mathrm{J} \cdot \mathrm{s}^{-1} \cdot \mathrm{sr}^{-1}\right)$ représente ce qui a été appelé pendant longtemps " l'intensité radiante "; mais il y a des raisons sérieuses pour éviter qu'un mot aussi généralement utilisé que "l'intensité " soit employé avec une signification aussi particulière. C'est pourquoi nous introduisons le terme "stérique " qui, ici, explicite l'opération $d / d \Omega$, laquelle donne la distribution par rapport à l'angle solide. Le flux stérique de particules (exprimé en $\mathrm{s}^{-1} \cdot \mathrm{sr}^{-1}$ ) est analogue.

La radiance (colonne 6) représente la caractérisation la plus compiète d'un faisceau d'une radiation monochromatique car elle représente la distribution par rapport à toutes les variables autres que la fréquence (surface perpendiculaire au faisceau, angle solide et temps).

Pour les rayonnements électromagnétiques, le quotient de la radiance par le carré de l'indice de réfraction est une quantité invariable lorsque le faisceau subit des modifications telles que la réflexion ou la réfraction en

( $\left.{ }^{1}\right)$ D'aucuns ont confondu cette grandeur avec celle que les radiobiologistes ont définie comme la « dose ". Bien que ces deux quantités aient les mêmes dimensions physiques, elles sont bien distinctes. La fluence est l'un des paramètres qui décrivent la radiation qui se propage au point considéré - d'où sa pertinence pour ce qui nous occupe ici. La dose, telle qu'elle a été définie par le Premier Congrès International de Photobiologie en 1954 et telle qu'elle a été utilisée depuis, est la quantité d'énergie qui est tombée sur l'unité de surface de l'objet irradié pendant le temps d'exposition. En général, cette dose a une valeur différente de celle de la fluence au même point. La seule exception a lieu lorsque la radiation provient d'une seule direction perpendiculaire à la surface considérée. Les termes ne sont pas synonymes. L'un n'est pas un remplacement récent de l'autre. Comme la " dose " n'intervient pas dans la description du champ de radiation, la discussion de ces points appartient à l'étape suivante dans le développement de cette nomenclature, et non pas à la présente étape.

VOL. $14-\mathrm{N}^{\circ} 2$ 
accord avec les lois de l'optique géométrique [2]. Aussi, en l'absence d'absorption et de diffusion, la radiance énergétique $\left(\mathrm{J} \cdot \mathrm{m}^{-2} \cdot \mathrm{s}^{-1} \cdot \mathrm{sr}^{-1}\right)$ et la radiance particulaire (photonique) $\left(\mathrm{m}^{-2} \cdot \mathrm{s}^{-1} \cdot \mathrm{sr}^{-1}\right)$ ont des valeurs constantes pour tous les points qui se trouvent le long d'un parcours lumineux où l'indice de réfraction est le même. Si la valeur de cette fonction de distribution est connue avec suffisamment de détails, les valeurs de toutes les autres caractéristiques du champ peuvent être obtenues par son intégration.

Les grandeurs citées dans le tableau ne comprennent pas toutes les fonctions de distribution qui pourraient être considérées mais seulement celles qui, selon l'expérience, sont fréquemment utilisées pour décrire les champs de radiation. Ces grandeurs ont été soumises à l'A.I.P. lors du VII ${ }^{\mathbf{e}}$ Congrès International de Photobiologie à Rome le $1^{\text {er }}$ septembre 1976. Elles ont été approuvées par la majorité des présents à une exception près : le terme "fluence " a été désapprouvé parce que sa racine fluere = couler implique un courant, ou un flux, et non pas une densité d'énergie accumulée. Toutefois, aucun accord n'a été obtenu pour lui substituer un autre terme. Les auteurs de cet article seraient heureux de recevoir des critiques ou des commentaires de photochimistes et de photobiologistes sur ce point ou sur n'importe quel autre de ces propositions.

Les recommandations précédentes ne comprennent pas les grandeurs attachées à l'interaction d'un champ de radiation et de la matière. Si les conclusions dont il est fait ici état recueillent l'approbation des organismes de tutelle (et d'autres organismes concernés), les travaux seront poursuivis et un autre rapport présenté, afin de fournir une description et un schéma dosimétrique complets utilisés pour tous les types de radiations.

\section{BIBLIOGRAPHIE}

[1] Nicodemus F. E. Am J. Phys., 1963, 31, 368-377.

[2] Rupert C. S. Photochem. Photobiol., 1974, 20, 203-212. 